\title{
MEDICAL REVIEW
}

HONORARY EDITOR-IN-CHIEF

Qide Han, Peking University, Beijing, China

\section{COUNSELORS}

Jisheng Han, Peking University Health Science Center, Beijing, China Nanshan Zhong, Guangzhou Medical University, Guangzhou, China Yinglu Guo, Peking University Health Science Center, Beijing, China Youyou Tu, China Academy of Traditional Chinese Medicine, Beijing, China

\section{EDITOR-IN-CHIEF}

Qimin Zhan, Peking University, Beijing, China

\section{ASSOCIATE EDITORS-IN-CHIEF}

Cunyu Wang, University of California, Los Angeles, USA

Erdan Dong, Peking University Third Hospital, Beijing, China

Hongbing Shen, National Administration for Disease Control and Prevention, Beijing, China

Jie Qiao, Peking University, Beijing, China

Jun Wang, Peking University People's Hospital, Beijing, China

Lin Lu, Peking University Sixth Hospital, Beijing, China

Ning Zhang, Peking University Health Science Center, Beijing, China

Xiaosong Gu, Nantong University, Nantong, China

$\mathrm{Xin} L u$, Oxford University, England, UK

\section{MANAGING EDITORS}

Guifang Zeng, Peking University Health Science Center, Beijing, China Yangang Ren, Peking University Health Science Center, Beijing, China

\section{EDITORIAL BOARD MEMBERS}

Albert C. Ludolph, University of Ulm, Wuertenberg, Germany Baoguo Jiang, Peking University People's Hospital, Beijing, China Bo Huang, China Academy of Sciences, Beijing, China

Chen Wu, China Academy of Medical Sciences, Beijing, China Cheng Zhou, Peking University, Beijing, China

Chunli Song, Peking University Third Hospital, Beijing, China

Demin Zhou, Peking University, Beijing, China

Fuchou Tang, Peking University, Beijing, China

Hang Li, Peking University First Hospital, Beijing, China

Hattori Nobutaka, Juntendo University, Japan

Hongqiang Sun, Peking University Sixth Hospital, Beijing, China Hui Wang, Peking University People's Hospital, Beijing, China Jiafu Ji, Beijing Cancer Hospital, Beijing, China Joseph Kolars, University of Michigan, Michigan, United States Jun Guo, Beijing Cancer Hospital, Beijing, China

Kan Gong, Peking University First Hospital, Beijing, China Li Yang, Peking University First Hospital, Beijing, China Liangyi Chen, Peking University, Beijing, China Liming Li, Peking University, Beijing, China Lunquan Sun, Central South University, Changsha, China Min Ye, Peking University, Beijing, China
Ming $X u$, Peking University Third Hospital, Beijing, China Musheng Zeng, Sun Yat-sen University, Guangzhou, China Nigel Hooper, The University of Manchester, Manchester, United Kingdom Ogawa Hideoki, Juntendo University, Japan Pengfei Tu, Peking University, Beijing, China Qiang Zhang, Peking University, Beijing, China Qingyue Meng, Peking University, Beijing, China Qunying Lei, Fudan University, Shanghai, China Siyan Zhan, Peking University, Beijing, China Wei Kong, Peking University, Beijing, China Weihua Yue, Peking University Sixth Hospital, Beijing, China Xiaojun Huang, Peking University People's Hospital, Beijing, China Xuliang Deng, Peking University School of Stomatology, Beijing, China Yali Cong, Peking University, Beijing, China Yining Huang, Peking University First Hospital, Beijing, China Yongsheng Zhou, Peking University Hospital of Stomatology, Beijing, China You Wan, Peking University, Beijing, China Yuxin Yin, Peking University, Beijing, China Zemin Zhang, Peking University, Beijing, China Zhanguo Li, Peking University People's Hospital, Beijing, China

\section{DE GRUYTER}


Medical Review (MR) is a peer reviewed open access journal affiliate to Peking University Health Science Centre. MR publishes high-quality internationally competitive review articles on cutting-edge progress and achievement from various areas of medical science, specifically focuses on clinical medicine, basic medicine, preventive medicine, medical ethics and multidisciplinary and emerging topics. MR is also committed to promote fundamental benefits among medical research communities, decision-makers and the public by conscientiously reviewing strategical and macro-level policies on medical-related issues and public health.

\section{SPECIAL STATEMENT}

All articles published in this journal represent the views of the authors and do not reflect any official policy of the Medical Review. Authors are solely responsible for their manuscripts and data published in the journals and any mistakes in the published articles. Publication does not constitute endorsement by the journal. No part of the journal should be reproduced or transmitted in any form or by any means without written permission from the journal.

ISSN: 2749-9642

Print ISSN: 2097-0733 (applies for copies in China)

CN: CN 10-1793/R

ONLINE SUBMISSION

https://mc.manuscriptcentral.com/mr

All information regarding notes for contributors, online submission, open access, back volumes and orders is available online at www.degruyter.com/mr or www.mrjournal.org

EDITOR-IN-CHIEF Qimin Zhan, Peking University, Beijing, China

EDITORIAL OFFICE No. 38 Xueyuan Road, Haidian District, Beijing 100191, China. Tel.: +86 (010) 828055 81, E-mail: mr@ mrjournal.org

RESPONSIBLE INSTITUTION Ministry of Education People's Republic of China

SPONSOR Peking University, No. 5, Yiheyuan Road, Haidian District, Beijing

JOURNAL COORDINATOR Alexander Görlt, De Gruyter, Genthiner Str. 13, 10785 Berlin, Germany. Tel.: +49 3026005234 ,

E-mail: alexander.goerlt@degruyter.com

RESPONSIBLE FOR ADVERTISEMENTS Markus Kügel, De Gruyter, Rosenheimer Str. 143, 81671 München, Germany.

Tel.: +498976902 424, E-mail: anzeigen@ degruyter.com

TYPESETTING TNQ Technologies, Chennai, India

PRINTING Beijing Shengpin Fashion Technology Development Co., Ltd.

Published by Walter de Gruyter GmbH on behalf of

(C) 2022 Peking University Health Science Center, China

Offenlegung der Inhaber und Beteiligungsverhältnisse gem. § 7a Abs. 1 Ziff. 1, Abs. 2 Ziff. 3 des Berliner Pressegesetzes: Die Gesellschafter der Walter de Gruyter GmbH sind: Cram, Gisela, Rentnerin, Berlin; Cram, Elsbeth, Pensionärin, Rosengarten-Alvesen; Cram, Dr. Georg-Martin, UnternehmensSystemberater, Stadtbergen; Cram, Maike, Wien (Österreich); Cram, Jens, Mannheim; Cram, Ingrid, Betriebsleiterin, Tuxpan/Michoacan (Mexiko); Cram, Sabina, Mexico, DF (Mexiko); Cram, Silke, Wissenschaftlerin, Mexico DF (Mexiko); Cram, Björn, Aachen; Cram, Berit, Hamm; Cram-Gomez, Susana, Mexico DF (Mexiko); Cram-Heydrich, Walter, Mexico DF (Mexico); Cram-Heydrich, Kurt, Angestellter, Mexico DF (Mexico); Duvenbeck, Brigitta, Oberstudienrätin i.R., Bad Homburg; Gädeke, Gudula, M.A., Atemtherapeutin/Lehrerin, Tübingen; Gädeke, Martin, Student, Ingolstadt; Gomez Cram, Arturo Walter, Global Key Account Manager, Bonn, Gomez Cram, Ingrid Arlene, Studentin, Mexico, DF (Mexiko), Gomez Cram, Robert, Assistant Professor, London UK, Lubasch, Dr. Annette, Ärztin, Berlin; Schütz, Dr. Christa, Ärztin, Mannheim; Schütz, Sonja, Diplom.-Betriebswirtin (FH), Berlin; Schütz, Juliane, Berlin; Schütz, Antje, Berlin; Schütz, Valentin, Mannheim; Seils, Dorothee, Apothekerin, Stuttgart; Seils, Gabriele, Journalistin, Berlin; Seils, Christoph, Journalist, Berlin; Siebert, John-Walter, Pfarrer, Oberstenfeld; Anh Vinh Alwin Tran, Student, Zürich (Schweiz), Tran, Renate, Mediatorin, Zürich (Schweiz); Carmen Siebert, Angestellte, Kempten; Duyen Anh Johann Tran, Architekt, Zürich (Schweiz); Lang Lieu Laurin Tran, Lehrperson, Zürich (Schweiz); Duyen Mai Marie Tran, Studentin, Zürich (Schweiz); Anh Huy Merlin Tran, Schüler, Zürich (Schweiz) 


\section{Contents}

\section{Preface}

Qide Han

New challenges in medicine -1

\section{Inaugural Editorial}

Qimin Zhan

Welcome to your new journal: Medical Review -2

\section{Editorial}

Erdan Dong

Cell-cell crosstalk in the heart - 3

\section{Perspectives}

Jun Zhou, Zhuoying Ren, Jie Xu, Jifeng Zhang and Y. Eugene Chen

Gene editing therapy ready for cardiovascular diseases: opportunities, challenges, and perspectives -6

Hui Gong, Ting Wang and Qingbo Xu

Resident stem cells in the heart -10

Yajing Wang, Wayne Bond Lau and Xinliang Ma

"Know Diabetes by Heart": role of adipocyte-

cardiomyocyte communications -14

\section{Reviews}

Yang Li, Xiaoxiang Tian, Quanyu Zhang, Chenghui Yan and Yaling Han

A novel function of CREG in metabolic disorders - 18

Chak Kwong Cheng and Yu Huang

The gut-cardiovascular connection: new era for

cardiovascular therapy -23

Xiaojun Du

Sympatho-adrenergic mechanisms in heart failure: new insights into pathophysiology -47

Jinlong He, Zhen Cui and Yi Zhu

The role of caveolae in endothelial dysfunction -78

George Liu, Pingping Lai, Jiabao Guo, Yuhui Wang and Xunde Xian

Genetically-engineered hamster models: applications and perspective in dyslipidemia and atherosclerosis-related cardiovascular disease -92 